Correspondence Leonard H. Damelin leonardd@nicd.ac.za

Received 15 July 2010

Accepted 26 October 2010

\section{Identification of predominant culturable vaginal Lactobacillus species and associated bacteriophages from women with and without vaginal discharge syndrome in South Africa}

\author{
Leonard H. Damelin, ${ }^{1}$ Maria Paximadis, ${ }^{1}$ Demetra Mavri-Damelin, ${ }^{2}$ \\ Monica Birkhead, ${ }^{3}$ David A. Lewis ${ }^{4,5}$ and Caroline T. Tiemessen ${ }^{1}$
}
${ }^{1}$ AIDS Virus Research Unit, National Institute for Communicable Diseases (NHLS), Private Bag X4, Sandringham 2131, Johannesburg, South Africa
${ }^{2}$ School of Molecular and Cell Biology, University of the Witwatersrand, Johannesburg 2050, South Africa
${ }^{3}$ Electron Microscope Unit, National Institute for Communicable Diseases (NHLS), Private Bag X4, Sandringham 2131, Johannesburg, South Africa
${ }^{4}$ Sexually Transmitted Infections Reference Centre, National Institute for Communicable Diseases (NHLS), Private Bag X4, Sandringham 2131, Johannesburg, South Africa
${ }^{5}$ Department of Internal Medicine, Faculty of Health Sciences, University of the Witwatersrand, Johannesburg 2050, South Africa

\begin{abstract}
Lactobacillus jensenii, Lactobacillus crispatus, Lactobacillus iners, Lactobacillus gasseri and Lactobacillus vaginalis were identified by $16 \mathrm{~S}$ rRNA gene sequencing as the predominant culturable vaginal Lactobacillus species in a group of South African women, comprising 24, 22, 10 , 10 and $9 \%$, respectively. A significant effect of vaginal discharge syndrome (VDS) and bacterial vaginosis (BV) on the distribution of predominant Lactobacillus species was observed. Whilst $L$. crispatus isolates were almost equally distributed between individuals with and without VDS and were not significantly reduced in women with BV versus normal microflora, $L$. jensenii isolates were significantly reduced in women with VDS $(P=0.022)$ and reduced in women with BV versus normal microflora $(P=0.053)$. Unlike $L$. crispatus, $L$. jensenii isolates were also significantly reduced in women with BV-associated VDS versus women without VDS and with normal microflora $(P=0.051)$. In addition, lysogeny was commonly observed for $L$. crispatus, with $77 \%$ of isolates yielding phage particles with contractile and non-contractile tails. Only $29 \%$ of $L$. jensenii isolates yielded phage particles, and these were visible as tailless or podo-like particles.
\end{abstract}

\section{INTRODUCTION}

Vaginal mucosal microfloras are typically dominated by Gram-positive Lactobacillus species, which serve as an important natural barrier against infection (Vásquez et al., 2002; Iqbal \& Kaul, 2008). The colonization of vaginal mucosa by engineered microbicide-secreting Lactobacillus strains is therefore seen as an economical and long-lasting means of enhancing this natural mucosal barrier for the prevention of human immunodeficiency virus (HIV) infection via vaginal intercourse (Liu et al., 2006, 2008). However, to avoid potentially undesirable perturbations of the host's existing microflora by the introduction of exogenous Lactobacillus strains into the cervico-vaginal

Abbreviations: BV, bacterial vaginosis; VDS, vaginal discharge syndrome. mucosa, we have suggested that an alternative and potentially less disruptive strategy could be utilized. We have proposed that anti-HIV molecules could be expressed within the cervico-vaginal milieu by endogenous vaginal Lactobacillus populations that have been engineered in situ via plasmid transduction. To this end, we have recently successfully established that a predominant vaginal Lactobacillus species, Lactobacillus gasseri, can be transduced at high frequency, with $\Phi$ adh-derived transducing particles, resulting in transductants that stably express and secrete the HIV co-receptor antagonists CCL5 and CCL3 (Damelin et al., 2010), thus supporting the use of transduction of endogenous vaginal Lactobacillus species as a potential strategy for the prevention of HIV infection across mucosal membranes. Encouraged by our findings, we have now sought to identify and develop novel 
transduction models for predominant vaginal Lactobacillus species within the South African population. Therefore, in this study, we isolated and characterized the predominant culturable vaginal Lactobacillus species and associated bacteriophages from pre-menopausal South African women and investigated the effect of vaginal discharge syndrome (VDS) and bacterial vaginosis (BV) on the distribution of predominant Lactobacillus species. To our knowledge, this is the first time that such a study has been undertaken in South Africa.

\section{METHODS}

Specimen collection. Vaginal swabs were collected from 144 premenopausal volunteers aged 18 and over, with a median age of 32 years, with $(n=75)$ or without $(n=69)$ VDS. VDS, as defined by the World Health Organization and South African syndromic management guidelines, is abnormal vaginal discharge, which may be accompanied by dysuria, vulval itching or vulval burning (WHO, 2003). Consecutive consenting women were recruited at two public service outpatient clinics in Johannesburg, South Africa, by clinical staff of the Sexually Transmitted Infections Reference Centre (NICD/ NHLS). The study, including specimen collection, was approved by the University of the Witwatersrand's Human Research Ethics Committee (Medical) (protocol no. M080988). Vaginal swabs were used initially to produce a vaginal smear for subsequent Gram staining before being placed in anaerobic transport tubes (BBL Vacutainer Anaerobic Specimen Collectors; BD).

Bacterial culture. Upon arrival at the laboratory, on the same day or $18 \mathrm{~h}$ after collection swabs were streaked onto Rogosa, deManRogosa-Sharpe (MRS) and Columbia agar plates with $5 \%$ defibrinated sheep blood. Plates were incubated anaerobically for $48 \mathrm{~h}$ at $37{ }^{\circ} \mathrm{C}$. Representative colonies were Gram-stained and those yielding Gram-positive/-variable rods were used to inoculate MRS broth or Lactobacillus iners broth (for colonies obtained from blood agar; ATCC Broth recipe no. 1685). Glycerol stocks (15\%) of culturable isolates were stored at $-80{ }^{\circ} \mathrm{C}$ until further use.

Determination of BV. The Gram-stained vaginal smears obtained above were scored according to the method of Nugent et al. (1991) to detect normal microflora [Nugent score (NS) 0-3], intermediate flora (NS 4-6) and BV (NS 7-10).

$16 S$ rRNA gene sequencing. Isolates were identified to the species level using $16 \mathrm{~S}$ rRNA gene sequencing as described previously (Yu et al., 2009). Sequences were subjected to nucleotide-nucleotide BLAST and compared to known 16S rRNA genes in the public databases for species identification. The following 22 Lactobacillus ATCC reference strains were used to validate this method: L. acidophilus ATCC 4357; L. brevis ATCC 14869; L. buchneri ATCC 4005; L. casei subsp. casei ATCC 393; L. crispatus ATCC 33197; L. crispatus ATCC 33820; L. delbrueckii subsp. lactis ATCC 4797; L. delbrueckii subsp. lactis ATCC 12315; L. fermentum ATCC 11739; L. fermentum ATCC 14931; L. gasseri ATCC 9857; L. iners ATCC 55195; L. jensenii ATCC 25258; L. johnsonii ATCC 33200; L. minutus ATCC 33267; L. paracasei subsp. paracasei ATCC 27216; L. plantarum ATCC 14917; L. reuteri ATCC 23272; L. rhamnosus ATCC 21052; L. ruminis ATCC 25644, L. salivarius subsp. salicinius ATCC 11742; and L. vaginalis ATCC 49540.

Isolation of bacteriophages and electron microscopy. Overnight MRS broth cultures of L. crispatus and $L$. jensenii isolates were diluted to an $\mathrm{OD}_{590}$ of 0.1 , induced with $0.4 \mu \mathrm{g}$ mitomycin $\mathrm{C} \mathrm{ml}^{-1}$ for $18 \mathrm{~h}$ and centrifuged at $3000 \mathrm{~g}$ for $30 \mathrm{~min}$. Putative induced phages in the resultant supernatants were precipitated with polyethylene glycol and pelleted by centrifugation, as described previously (Damelin et al., 2010). Resuspended putative phage pellets were placed on Formvarcoated copper grids, negatively stained with $2 \%$ phosphotungstic acid, viewed with a transmission electron microscope at $80 \mathrm{kV}$ [FEI Tecnai Biotwin Spirit TEM $(120 \mathrm{kV})]$ and photographed with a MegaView III camera.

Statistical analysis. Associations were analysed using Fisher's exact test, with the level of statistical significance set at $P \leqslant 0.05$.

\section{RESULTS}

From 144 swabs, 115 isolates were cultured, with $36 \%$ of individuals (52/144) yielding culturable lactobacilli and six individuals yielding two culturable Lactobacillus species (Table 1). Lactobacillus species made up $50 \%$ of the culturable isolates, of which $L$. jensenii and $L$. crispatus were the predominant species ( 24 and $22 \%$, respectively; Table 1). L. iners and L. gasseri were also prevalent, each making up $10 \%$ of the total Lactobacillus isolates obtained, whilst L. vaginalis contributed $9 \%$ of the total lactobacilli (Table 1). Of the 57 non-Lactobacillus culturable bacteria, Gardnerella vaginalis was predominant $(22 \%$ of total isolates and $44 \%$ of non-Lactobacillus isolates), whilst

Table 1. Predominant vaginal bacterial species from South African adult females

\begin{tabular}{|lc|}
\hline Species & $\begin{array}{c}\text { No. of individuals with the } \\
\text { indicated species* }\end{array}$ \\
\hline Lactobacillus acidophilus & 1 \\
Lactobacillus casei & 1 \\
Lactobacillus coleohominus & 1 \\
Lactobacillus crispatus & 13 \\
Lactobacillus gasseri & 6 \\
Lactobacillus iners & 6 \\
Lactobacillus ingluviei & 1 \\
Lactobacillus jensenii & 14 \\
Lactobacillus mucosae & 3 \\
Lactobacillus murinus & 1 \\
Lactobacillus oris & 1 \\
Lactobacillus reuteri & 2 \\
Lactobacillus salivarius & 3 \\
Lactobacillus vaginalis & 5 \\
Streptococcus anginosus & 14 \\
Gardnerella vaginalis & 25 \\
Actinomyces urogenitalis & 2 \\
Staphylococcus epidermidis & 1 \\
Atopobium vaginae & 1 \\
Aerococcus christensenii & 1 \\
Unidentifiable vaginal species & 11 \\
Unidentifiable Gardnerella species & 2 \\
& \\
\hline
\end{tabular}

${ }^{*}$ Six individuals harboured two Lactobacillus species: one individual had $L$. crispatus and L. iners, one had L. crispatus and L. salivarius, three had L. crispatus and L. jensenii, and one had L. jensenii and $L$. salivarius. 
Table 2. Effect of Nugent score (NS) and VDS on Lactobacillus isolate frequency, and distribution of phage-yielding isolates

Numbers in parentheses represent the number of phage-yielding isolates for each category.

\begin{tabular}{|c|c|c|c|c|c|}
\hline \multirow[t]{2}{*}{ Species } & \multicolumn{3}{|c|}{ No. of individuals yielding the indicated species with NS of: } & \multicolumn{2}{|c|}{$\begin{array}{l}\text { No. of individuals yielding the indicated } \\
\text { species with or without VDS }\end{array}$} \\
\hline & 0-3 & $4-6$ & $7-10$ & VDS & No VDS \\
\hline L. acidophilus & 1 & & & & 1 \\
\hline L. casei & 1 & & & & 1 \\
\hline L. coleohominus & & 1 & & 1 & \\
\hline L. crispatus & $9(6)$ & $1(1)$ & $3(3)$ & $6(5)$ & $7(5)$ \\
\hline L. gasseri & 2 & 4 & & 2 & 4 \\
\hline L. iners & 6 & & & 3 & 3 \\
\hline L. ingluviei & 1 & & & & 1 \\
\hline L. jensenii & $11(3)$ & $2(1)$ & 1 & 3 & $11(4)$ \\
\hline L. mucosae & 2 & & 1 & 2 & 1 \\
\hline L. murinus & & 1 & & 1 & \\
\hline L. oris & 1 & & & 1 & \\
\hline L. reuteri & 1 & 1 & & 2 & \\
\hline L. salivarius & 3 & & & 1 & 2 \\
\hline L. vaginalis & 2 & 1 & 2 & 2 & 3 \\
\hline
\end{tabular}

Streptococcus anginosus accounted for $12 \%$ of total isolates and $25 \%$ of non-Lactobacillus isolates (Table 1 ).

Microscopy of the vaginal smears indicated that $48 \%$ of women had normal microflora, $26 \%$ had intermediate flora and $26 \%$ had BV. Unsurprisingly, $69 \%$ of culturable Lactobacillus species were obtained from individuals with normal microflora, whilst 19 and $12 \%$ of Lactobacillus species were obtained from individuals with intermediate flora and BV, respectively (Table 2), with L. crispatus accounting for $43 \%$ of lactobacilli obtained from individuals with BV (Table 2). We did not observe any significant effect of HIV status on the abundance or distribution of Lactobacillus species within the cohort; however, we did observe a significant effect of VDS and BV on the distribution of predominant Lactobacillus species. Whilst L. crispatus isolates were almost equally distributed between individuals with and without VDS and were not significantly reduced in women with BV versus normal microflora, L. jensenii isolates were significantly reduced in women with $\operatorname{VDS}(P=0.022)$ and significantly reduced in women with $\mathrm{BV}$ versus those with normal microflora $(P=0.053)$ (Table 2). Furthermore, unlike L. crispatus, $L$. jensenii isolates were significantly reduced in women (a)

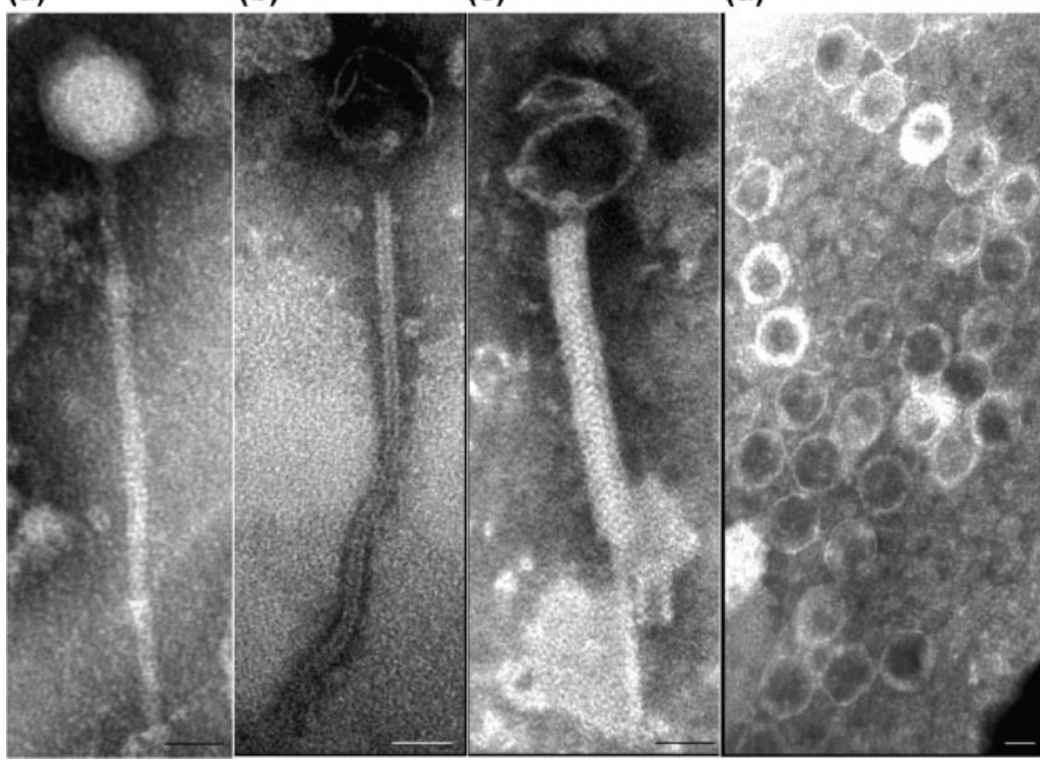

Fig. 1. Electron micrographs of bacteriophages from $L$. crispatus and $L$. jensenii isolates. ( $\mathrm{a}, \mathrm{b})$ Putative L. crispatus siphoviruses with long, non-contractile tails. (c) Putative L. crispatus myovirus with contractile tail. (d) Multiple capsid or podo-like particles observed for a representative $L$. jensenii isolate. Bars, $25 \mathrm{~nm}$. 
with BV-associated VDS versus women without VDS and with normal microflora $(P=0.051)$.

Next, we sought to identify endogenous phages in $L$. jensenii and L. crispatus, the predominant culturable vaginal Lactobacillus species identified. Lysogeny was commonly observed for L. crispatus with 10 of the 13 isolates $(77 \%)$ yielding phage particles with contractile and non-contractile tails (Fig. 1a-c). Only four of the 14 L. jensenii isolates $(29 \%)$ yielded phage particles; these were visible as tailless or podo-like particles (Fig. 1d).

\section{DISCUSSION}

Our findings indicated that the culturable vaginal Lactobacillus species predominant among women in South Africa (L. crispatus, L. gasseri, L. iners and L. jensenii) are similar to those found to predominate in American and European studies (Antonio et al., 1999; Kiliç et al., 2001; Vásquez et al., 2002; De Backer et al., 2007).

We also observed an apparent increase in persistence for $L$. crispatus isolates over $L$. jensenii isolates in individuals with $\mathrm{BV}$ or VDS, as the former were isolated in equal frequency from individuals with VDS and were the most frequent isolates in individuals with $\mathrm{BV}$, whilst $L$. jensenii isolates were significantly less recoverable from individuals with VDS and BV than those without. As a high degree of lysogeny $(77 \%)$ was observed for vaginal $L$. crispatus strains, and as it has been established that prophages contribute directly to host survival in unfavourable environments by conferring survival phenotypes to lysogens (Chibani-Chennoufi et al., 2004; Paul, 2008; Schuch \& Fischetti, 2009), it is tempting to speculate that some of the mechanisms that allow the persistence of $L$. crispatus strains within the perturbed vaginal milieu of individuals with VDS and BV may be phage-conferred. In support of this speculation is the finding that Streptococcus pneumoniae lysogens harbouring the MM1 phage exhibit improved adherence to epithelial cells, potentially allowing increased persistence within the nasopharynx (Loeffler \& Fischetti, 2006). However, further research would be required to determine whether analogous phage-conferred phenotypes exist for vaginal $L$. crispatus strains. In any event, our findings suggest that, due to its high frequency of lysogeny and persistence within individuals with VDS and BV, $L$. crispatus represents the most favourable vaginal Lactobacillus isolate for the development of novel transduction models specific for the South African population.

\section{ACKNOWLEDGEMENTS}

This work was funded by a grant from the Bill \& Melinda Gates Foundation through the Grand Challenges Explorations Initiative (Grant \# 51825) (to L. H. D.).

\section{REFERENCES}

Antonio, M. A. D., Hawes, S. E. \& Hillier, S. L. (1999). The identification of vaginal Lactobacillus species and the demographic and microbiologic characteristics of women colonized by these species. J Infect Dis 180, 1950-1956.

Chibani-Chennoufi, S., Bruttin, A., Dillmann, M.-L. \& Brüssow, H. (2004). Phage-host interaction: an ecological perspective. J Bacteriol 186, 3677-3686.

Damelin, L. H., Mavri-Damelin, D., Klaenhammer, T. R. \& Tiemessen, C. T. (2010). Plasmid transduction using bacteriophage $\Phi$ adh for the expression of CC-chemokines by Lactobacillus gasseri ADH. Appl Environ Microbiol 76, 3878-3885.

De Backer, E., Verhelst, R., Verstraelen, H., Alqumber, M. A., Burton, J. P., Tagg, J. R., Temmerman, M. \& Vaneechoutte, M. (2007). Quantitative determination by real-time PCR of four vaginal Lactobacillus species, Gardnerella vaginalis and Atopobium vaginae indicates an inverse relationship between L. gasseri and L. iners. BMC Microbiol 7, 115-127.

lqbal, S. M. \& Kaul, R. (2008). Mucosal innate immunity as a determinant of HIV susceptibility. Am J Reprod Immunol 59, 44-54.

Kiliç, A. O., Pavlova, S. I., Alpay, S., Kiliç, S. S. \& Tao, L. (2001). Comparative study of vaginal Lactobacillus phages isolated from women in the United States and Turkey: prevalence, morphology, host range, and DNA homology. Clin Diagn Lab Immunol 8, 31-39.

Liu, X., Lagenaur, L. A., Simpson, D. A., Essenmacher, K. P., FrazierParker, C. L., Liu, Y., Tsai, D., Rao, S. S., Hamer, D. H. \& other authors (2006). Engineered vaginal Lactobacillus strain for mucosal delivery of the human immunodeficiency virus inhibitor cyanovirin-N. Antimicrob Agents Chemother 50, 3250-3259.

Liu, X., Lagenaur, L. A., Lee, P. P. \& Xu, Q. (2008). Engineering human vaginal Lactobacillus for surface expression of two-domain CD4. Appl Environ Microbiol 74, 4626-4635.

Loeffler, J. M. \& Fischetti, V. A. (2006). Lysogeny of Streptococcus pneumoniae with MM1 phage: improved adherence and other phenotypic changes. Infect Immun 74, 4486-4495.

Nugent, R. P., Krohn, M. A. \& Hillier, S. L. (1991). Reliability of diagnosing bacterial vaginosis is improved by a standardized method of Gram stain interpretation. J Clin Microbiol 29, 297-301.

Paul, J. H. (2008). Prophages in marine bacteria: dangerous molecular time bombs or the key to survival in the seas? ISME J 2, 579589.

Schuch, R. \& Fischetti, V. A. (2009). The secret life of the anthrax agent Bacillus anthracis: bacteriophage-mediated ecological adaptations. PLoS ONE 4, e6532-e6553.

Vásquez, A., Jakobsson, T., Ahrne, S., Forsum, U. \& Mölin, G. (2002). Vaginal Lactobacillus flora of healthy Swedish women. J Clin Microbiol 40, 2746-2749.

WHO (2003). Guidelines for the Management of Sexually Transmitted Infections. Geneva: World Health Organization. http://www.who.int/ entity/hiv/pub/sti/en/STIGuidelines2003.pdf

Yu, R. R., Cheng, A. T., Lagenaur, L. A., Huang, W., Weiss, D. E., Treece, J., Sanders-Beer, B. E., Hamer, D. H., Lee, P. P. \& other authors (2009). A Chinese rhesus macaque (Macaca mulatta) model for vaginal Lactobacillus colonization and live microbicide development. J Med Primatol 38, 125-136. 